\title{
On-line determination of serum propofol concentrations by expired air analysis
}

\author{
Eike Carstens • Andreas Hirn • Michael Quintel • \\ Jürgen Nolte • Melanie Jünger • Thorsten Perl • \\ Wolfgang Vautz
}

Received: 3 November 2009/Revised: 28 January 2010/Accepted: 2 February 2010 /Published online: 20 February 2010

(C) Springer-Verlag 2010

\begin{abstract}
Propofol (2,6-diisopropylphenol) - an intravenous anaesthetic - can be identified and quantified in expired air directly. For the first time, a $\beta$-radiation ion mobility spectrometer operated in the positive mode and coupled to a multi-capillary column for rapid (seconds-minutes) pre-separation (MCC/IMS) was used for the quantification of Propofol in expired air. The comparison of the concentrations in exhaled air $\left(300 \mathrm{ppt}_{\mathrm{V}}-5 \mathrm{ppb}_{\mathrm{V}}\right)$ and in serum $(0.3-5 \mu \mathrm{g} / \mathrm{mL})$ showed satisfying agreement affirmed by a correlation coefficient of 0.73 . Therefore, MCC/IMS is an adequate method to determine Propofol concentrations in exhaled air and may be applied for the prediction of venous concentrations or for automatic anaesthesia control.
\end{abstract}

Keywords Propofol ·2,6-diisopropylphenol - Anaesthesia · Serum · TIVA · Breath analysis · Ion mobility spectrometry · GC/MS $\cdot$ GC/IMS

\section{Introduction}

The monitoring of volatile anaesthetics in end-tidal breath is a standard procedure to improve safety of the patient and to adjust anaesthetic depth. Presently, this is not easy to perform for intravenous anaesthetics such as Propofol.

J. Nolte $\cdot$ M. Jünger $\cdot$ W. Vautz $(\bowtie)$

Metabolomics Department,

ISAS - Institute for Analytical Sciences,

Dortmund, Germany

e-mail: vautz@isas.de

E. Carstens $\cdot$ A. Hirn $\cdot$ M. Quintel $\cdot$ T. Perl

Department of Anaesthesiology, Emergency and Intensive Care Medicine, University of Göttingen,

Göttingen, Germany
Complex and time consuming off-line analyses of blood samples are required. Therefore, an on-line method for the determination of the serum anaesthetics concentration is desirable to improve safety and adjust anaesthetic depth or the level of sedation in intensive care units (ICU).

The on-line measurement of Propofol in exhaled air has already been demonstrated using different techniques like proton transfer reaction mass spectrometry (PTRMS) [1, 2], thermal desorption gas chromatography [3] and ion molecule reaction-mass spectrometry (IMR-MS) [4]. Those investigations showed close relations of Propofol concentrations in exhaled air and in blood [24]. However, those instruments are expensive and in general not commercially available as validated medical instrumentation.

For the present study, an ion mobility spectrometer in combination with a multi-capillary column (MCC/IMS) was applied for the quantification of Propofol in exhaled air for the first time as presented in [5] for the medical community, in particular with regard to the possible routine application. Here, the technical aspects will come to the fore. The results were compared to serum concentrations of Propofol determined by GC/MS. The MCC/IMS has already been applied successfully for medical purpose [68]. It is a suitable tool to analyse human breath as it provides high sensitivity (down to $\mathrm{ppt}_{\mathrm{V}}$ ) and selectivity combined with high-speed data acquisition (single spectra $0.1 \mathrm{~s} /$ complete breath analysis $\sim 5 \mathrm{~min}$ ) and relatively low technical expenditure.

\section{Experimental}

The bi-directional $\beta$-radiation MCC/IMS operated with synthetic air under ambient pressure and temperature used 
for the study was custom designed at ISAS and has been described in detail in literature as well as the software used for data acquisition and evaluation [6-8]. For preseparation, a $20 \mathrm{~cm}$ un-polar multi-capillary chromatographic column (OV-5, MULTICHROM, Novosibirsk, Russia) was operated isothermal at $40{ }^{\circ} \mathrm{C}$. Sampling was controlled by a $\mathrm{CO}_{2}$ sensor (IRMA, Fa. PhaseIn, Danderyd, Sweden), which was used to flush the sample loop ( $8 \mathrm{~mL}$ volume) only if carbon dioxide exceeded $25 \mathrm{mmHg}$, thus sampling only endtidal breath. The volume of the sample loop was then introduced directly into the MCC without any pre-treatment of the sample.

Propofol (2,6-diisopropylphenol) is characterised by a molecular mass of $178 \mathrm{u}$ and a phenolic chemical structure. Propofol and Thymol (used as internal standard) for calibration were obtained from Sigma-Aldrich (Seelze, Germany). A calibration gas generator (HovaCAL 3834SC VOC, Inspire Analytical Systems GmbH, Frankfurt am Main, Germany, see [9]) was used for calibration of MCC/IMS. The generator provided Propofol concentrations (1, 3, 5, 7, 9 and $\left.10 \mathrm{ppb}_{\mathrm{v}}\right)$ in humid air $\left(100 \%\right.$ relative humidity at $\left.37^{\circ} \mathrm{C}\right)$. The calibration results (correlation coefficient of 0.984 , $n=24$ ) are presented in Fig. 1. The Propofol concentration could be determined by the MCC/IMS in breath with a mean standard deviation of $2.5 \%$ and by GC/MS in serum with $12.2 \%$.

\section{Study population}

Thirteen patients scheduled for an elective ear-nose-throat surgery procedure (nine male, four female, all classified as American Society of Anaesthetist physical status I or II, see [10]) were involved in the study. In the mean, patients were

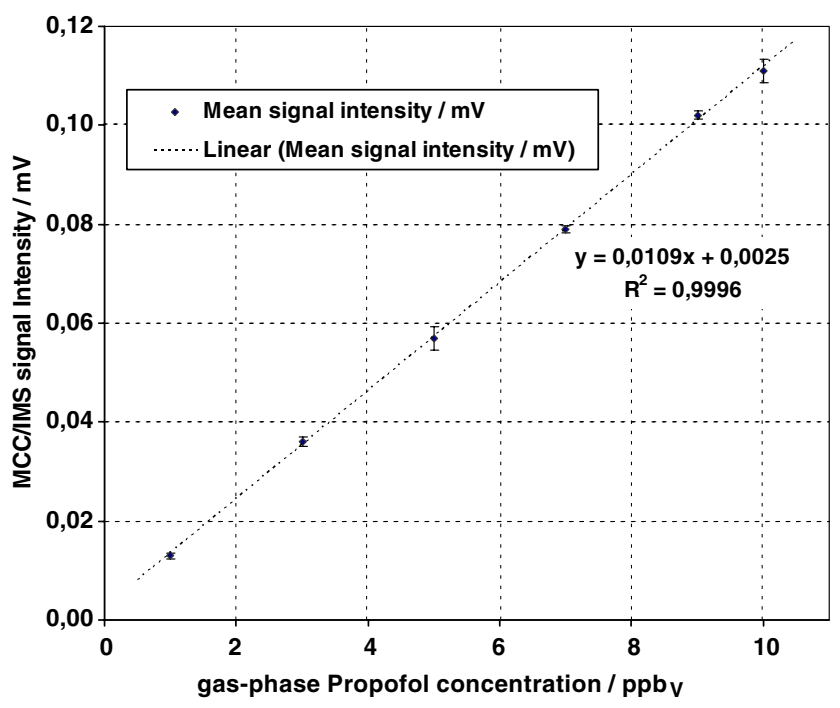

Fig. 1 Calibration of the MCC/IMS to gas-phase Propofol in humid air
45 years of age and their body-mass-index (BMI) was 24.6 (Table 1). The study was approved by the ethics committee of the University of Göttingen, Germany (protocol No. 01/ 07/08). All patients gave written informed consent to participate in the investigation.

Anaesthesia and sample acquisition

The patients received a standard total intravenous anaesthesia (TIVA) with Propofol (Disoprivan 1\%; Astra Zeneca, Wedel, Germany) as hypnotic agent, Remifentanil for analgesia and optional Rocuronium as muscle relaxant. Anaesthesia was induced with intravenous application of $2.1( \pm 0.7) \mathrm{mg} / \mathrm{kg}$ Propofol bolus and $30 \mu \mathrm{g} / \mathrm{kg} / \mathrm{h}$ Remifentanil. After tracheal intubation, the lung was ventilated with a standard anaesthesia respirator (Cato, Dräger, Lübeck, Germany). Propofol (3.9 $( \pm 1.8) \mathrm{mg} / \mathrm{kg} / \mathrm{h})$ and Remifentanil $(30 \mu \mathrm{g} / \mathrm{kg} / \mathrm{h})$ were applied continuously to maintain anaesthesia adjusted to clinical parameters. Sampling was performed as described above and in Total Intravenous Anaesthesia in analogy to Schubert et al. [11] measuring $\mathrm{CO}_{2}$ in breath with mainstream capnography (IRMA, Fa. PhaseIn, Danderyd, Sweden). Breath samples were drawn when the $\mathrm{CO}_{2}$ level exceeded $25 \mathrm{mmHg}$ and venous blood samples were obtained via a separate venous access at the opposite site of the drug infusion simultaneously. All samples were collected at steady state conditions (achieved by administration of Propofol and Remifentanil for $15 \mathrm{~min}$ ). Depending on the duration of surgery, one or two sample pairs of breath and serum were taken per patient.

\section{Determination of propofol concentrations by GC/MS}

Breath samples have been adsorbed on Tenax tubes $(1 \mathrm{~L}$ of breath, controlled by the sampling control of the $\mathrm{MCC} /$ IMS) and were thermally desorbed and analysed just as the blood samples (injected in one bolus at $250{ }^{\circ} \mathrm{C}$ ) using a GC/MS with EI-ionisation $(70 \mathrm{eV})$ by Agilent Technologies 6,890 N GC-system connected to an Agilent Technologies 5,973 mass selective detector (MSD; Gerstel, Mülheim, Germany). The operating parameters were: SSL-injector $180{ }^{\circ} \mathrm{C}$; column DB-5: $30 \mathrm{~m}$; 0,25 mm ID; 0,25 $\mu \mathrm{m}$ FD; $1 \mathrm{~mL} / \mathrm{min} \mathrm{He}$ const.; T-program $40{ }^{\circ} \mathrm{C} / 5{ }^{\circ} \mathrm{C} / \mathrm{min}-100{ }^{\circ} \mathrm{C} /$

Table 1 Demographics of the study population: Median (Min. Max.)

\begin{tabular}{|c|c|}
\hline Sex (male/female) & $9 / 4$ \\
\hline Age [years] & $45(1869)$ \\
\hline Weight [kg] & $76(58$ 107) \\
\hline Height $[\mathrm{cm}]$ & 173 (160 183) \\
\hline BMI & $25(19.638 .8)$ \\
\hline
\end{tabular}




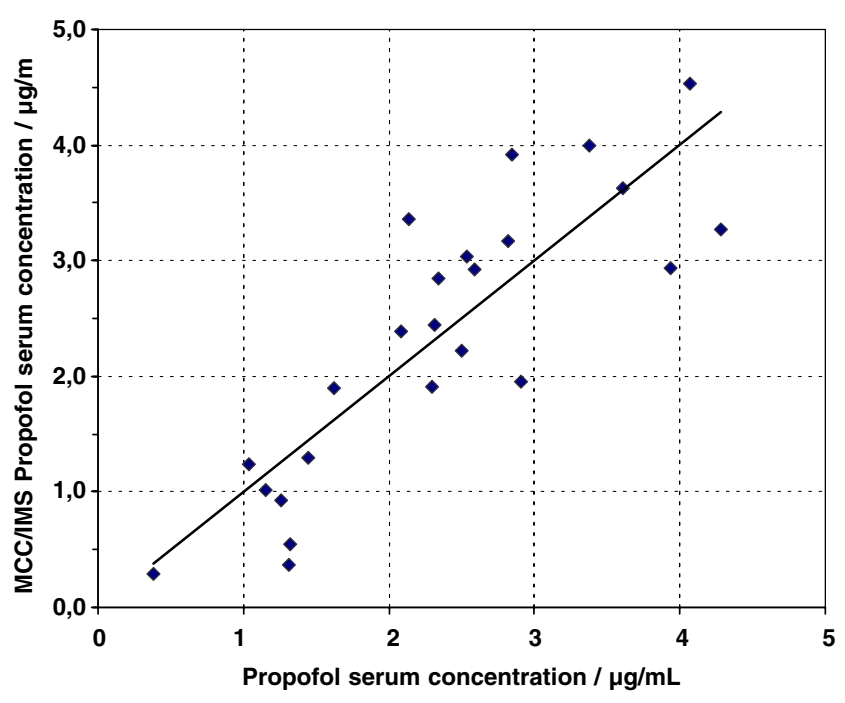

Fig. 2 Propofol concentrations in exhaled air determined by $\mathrm{MCC} /$ IMS and converted into serum concentrations versus Propofol serum concentrations determined by GC/MS. The solid line indicates a linaer regression with a correlation coefficient of 0.73

$20{ }^{\circ} \mathrm{C} / \mathrm{min}-200{ }^{\circ} \mathrm{C}(5 \mathrm{~min})$. Integrated signals from the internal standard Thymol ( $\mathrm{m} / \mathrm{z}$ 135,150) and from Propofol $(m / z 163,178)$ were evaluated by AMDIS/NIST (Automated Mass Spectral Deconvolution and Identification System; version 2.62, 2005; NIST version 2.0, 2005).

Simultaneously to breath sampling, venous blood was from the arm that was not used for the infusion. All samples were collected under steady-state conditions (Achieved by administration of Propofol and Remifentanil for $15 \mathrm{~min}$ ). Depending on the duration of surgery, 1-2 samples were obtained from each patient.

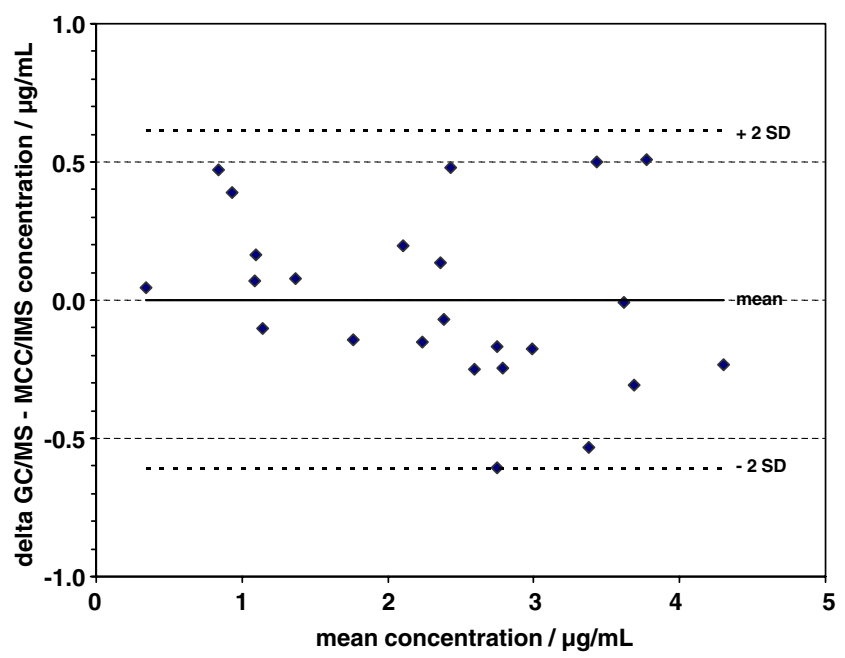

Fig. 3 Deviation of the MCC/IMS concentration from the mean concentration determined by both MCC/IMS and GC/MS. The dotted lines indicate twice the standard deviation

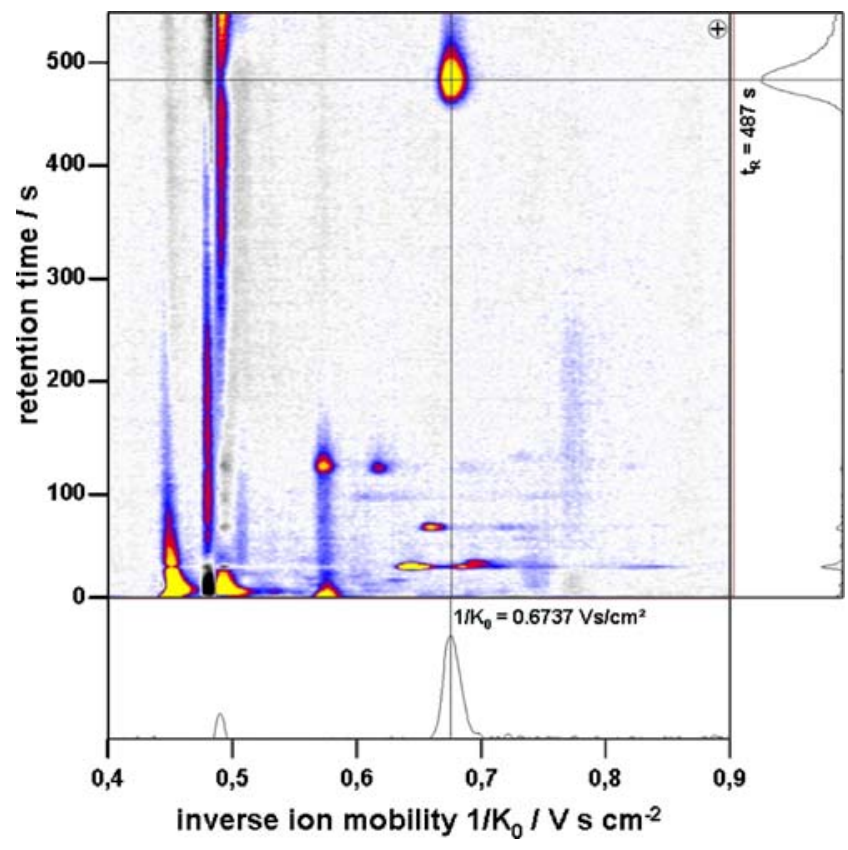

Fig. $4 \mathrm{MCC} / \mathrm{IMS}$ chromatogram of Propofol with pre-separation operated at $40{ }^{\circ} \mathrm{C}$. The Propofol signal is indicated by the cross line and the single spectra (bottom) and chromatogram (right) are displayed in the extra figures

\section{Results and discussion}

In total 24 measurements were carried out during anaesthesia of the study population. The Propofol signal in the MCC/IMS spectra was identified via parallel Tenax samples of breath and TD-GC/MS analysis earlier from a different study population. The Propofol concentration in exhaled air was determined by MCC/IMS and converted into serum concentrations. The related serum Propofol concentration was determined by GC/MS using the reference analyte for quantification. The comparison of both measurements is presented in Fig. 2. A correlation

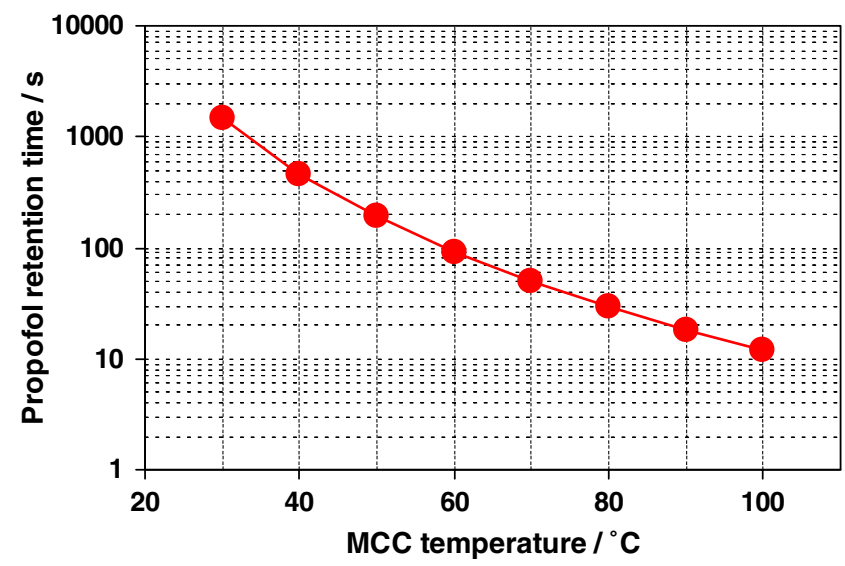

Fig. 5 From calibration measurements, a decrease of the Propofol retention time described by the formula $t_{\mathrm{R}}(T)=11^{8 *} T^{-3.98}$ was determined 
coefficient of 0.73 was obtained. In contrast to Miekisch et al. [12] but conformable to Grossherr et al. [3] a correlation between venous serum and exhaled air Propofol concentration was observed.

To provide more general information about the reliability of the serum Propofol concentration determined via breath analysis, the MCC/IMS concentrations were compared to the mean concentration as determined by both MCC/IMS and GC/MS (see Fig. 3). The standard deviation of the MCC/IMS compared to this normalised mean value was with $12.3 \%$ in the same range as found when other techniques are used [2-4].

For the present study, the pre-separation of the MCC/ IMS was operated at $40{ }^{\circ} \mathrm{C}$. With this experimental setup, the Propofol signal was detected with a mean retention time of $474.5 \mathrm{~s}$ and a mean inverse reduced ion mobility $1 / \mathrm{K}_{0}$ of $0.6737 \mathrm{Vs} / \mathrm{cm}^{2}$ (see Fig. 4). However, as the signal occurs in general separated from others, the pre-separation temperature may be increased to reduce retention time and analysis time respectively.

Calibration measurements at different MCC temperatures showed, that retention time follows an almost logarithmic decrease with increasing MCC temperature. For Propofol, the correlation can be described by the formula $t_{R}(T)=1.1 * 10^{8 *} T^{-3.98}$ (see Fig. 5). Exceeding $70{ }^{\circ} \mathrm{C}$, retention time is already $<1 \mathrm{~min}$ and at $100{ }^{\circ} \mathrm{C}$ only $10 \mathrm{~s}$. The very isolation location of the Propofol signal in the breath spectra would furthermore enable to use a $10 \mathrm{~cm}$ MCC instead of $20 \mathrm{~cm}$, which will halve the retention time. Therefore, at $100{ }^{\circ} \mathrm{C}$, the retention time of Propofol concentration can be determined after about $5 \mathrm{~s}$.

\section{Conclusion}

The correlation between exhaled air concentrations and serum concentrations of Propofol was described for the first time using a MCC/IMS. The system obtains a point of care measurement with non-invasive sampling and reliable prediction of venous Propofol concentrations without preconcentration. The on-line determination (5-10 s after sample acquisition) of serum Propofol concentrations via breath analysis with regard to safety factors is feasible for infusion control at ICU and for Total Intravenous Anaesthesia (TIVA).
Acknowledgements The financial support of the Bundesministerium für Bildung und Forschung and the Ministerium für Wissenschaft und Forschung des Landes Nordrhein-Westfalen is gratefully acknowledged. The dedicated work of Luzia Seifert, Susanne Krois, Stefanie Güssgen, Sandra Stelzer and Jacqueline Friedrich, technicians at ISAS, was indispensable for the successful investigations. Furthermore, the valuable contribution of Bertram Boedeker related to data evaluation is acknowledged as well as the general support of B\&S Analytik, Dortmund, Germany.

\section{References}

1. Harrison GR, Critchley AD, Mayhew CA, Thompson JM (2003) Real-time breath monitoring of propofol and its volatile metabolites during surgery using a novel mass spectrometric technique: a feasibility study. Br J Anaesth 91:797-799

2. Takita A, Masui K, Kazama T (2007) On-line monitoring of end-tidal propofol concentration in anesthetized patients. Anesthesiology 106:659-664

3. Grossherr M, Hengstenberg A, Meier T, Dibbelt L, Gerlach K, Gehring H (2006) Discontinuous monitoring of propofol concentrations in expired alveolar gas and in arterial and venous plasma during artificial ventilation. Anesthesiology 104:786-790

4. Hornuss C, Praun S, Villinger J, Dornauer A, Moehnle P, Dolch M, Weninger E, Chouker A, Feil C, Briegel J, Thiel M, Schelling G (2007) Real-time monitoring of propofol in expired air in humans undergoing total intravenous anesthesia. Anesthesiology 106:665-674

5. Perl T, Carstens E, Hirn A, Quintel M, Vautz W, Nolte J, Jünger M (2009) Determination of serum propofol concentrations by breath analysis using ion mobility spectrometry. Br J Anaesth 103 (6):822-827

6. Westhoff M, Litterst P, Freitag L, Baumbach JI (2007) Ion mobility spectrometry in the diagnosis of sarcoidosis: results of a feasibility study. J Physiol Pharmacol 58(Suppl 5):739-751

7. Ruzsanyi V, Sielemann S, Baumbach JI (2005) Analysis of human breath using IMS. Int J Ion Mobil Spectrom 8:5-7

8. Vautz W, Nolte J, Fobbe R, Baumbach JI (2009) Breath analysisperformance and potential of ion mobility spectrometry. J Breath Res 2009. doi:10.1088/1752-7155/3/3/036004

9. Vautz W, Schmäh M (2009) HovaCAL ${ }^{\circledR}$ —a generator for multicomponent humid calibration gases. Int J Ion Mobil Spectrom 12:139-147

10. Owens WD, Felts JA, Spitznagel EL Jr (1978) ASA physical status classifications: a study of consistency of ratings. Anesthesiology 49:239-243

11. Schubert J, Spittler KH, Braun G, Geiger K, Guttmann J (2001) $\mathrm{CO} 2$-controlled sampling of alveolar gas in mechanically ventilated patients. Appl Physiol 90:486-492

12. Miekisch W, Fuchs P, Kamysek S, Neumann C, Schubert JK (2008) Assessment of propofol concentrations in human breath and blood by means of HS-SPME-GC-MS. Clin Chim Acta 395:32-37 